\title{
An Elementary Remark on Maximal Gaps Between Successive Primes
}

\author{
By Selmer M. Johnson
}

1. Introduction. In a recent note [1], Shanks discussed the problem of estimating upper bounds to the location of strings of $g$ consecutive composite integers. $\mathrm{He}$ defined $p(g)$ to be the first prime following such a string. Using the well-known Lucas argument, he showed that

$$
\log p(g)<g \log g
$$

for sufficiently large $g$. Next he used an elegant form of the prime-number theorem due to Rosser and Schoenfeld [2] to obtain a sharper result,

$$
\log p(g)<g+\frac{5}{2}
$$

for $p \geqq 67$.

He pointed out that many authors using more difficult arguments have obtained slightly better results, but these are still much too high compared to a conjectured asymptote:

$$
\log p(g) \sim \sqrt{ } g,
$$

that is based on a heuristic argument and numerical evidence which he presented.

The purpose of the present note is to show that simple elementary arguments lead to a bound for $\log p(g)$ which is essentially half of that in (2). This is not as good as some of the difficult results mentioned above but may be worth noting because of its constructive simplicity.

2. The Improved Elementary Upper Bound for $p(g)$.

Theorem. Let $p_{i}$ be the ith prime. If $p_{n} \leqq g / 2<p_{n+1}<p_{n+2}$, then

$$
p(g)<\prod_{i=1}^{n+2} p_{i}
$$

Proof. First observe that for $A, B, C$, pairwise relatively prime integers, there is some positive integer $t<B C$ such that $t A \equiv-1(\bmod B)$ and $t A \equiv+1(\bmod$ $C)$. Then $(B C-t) A \equiv+1(\bmod B)$ and $(B C-t) A \equiv-1(\bmod C)$.

Iet

$$
A=\prod_{i=1}^{n} p_{i}, \quad B=p_{n+1}, \quad C=p_{n+2} .
$$

For some $t<B C / 2$ we have the set

$$
t A, \quad t A \pm 1, \quad t A \pm 2, \quad t A \pm 3, \quad t A \pm 4, \cdots, t A \pm\left[\frac{g}{2}\right],
$$

forming a string of $1+2[g / 2] \geqq g$ consecutive composites for $g>9$. Then $p(g)<$ $2 t A$ by Bertrand's "Postulate," giving (4). For $g \leqq 9,(4)$ can be verified directly. Corollary (NOT ELEMENTARY).

Received March 5, 1965. 


$$
\log p(g)<\theta\left(\frac{g}{2}\right)+\log p_{n+1} \cdot p_{n+2}<\frac{g}{2}\left(1+\frac{1}{2 \log \frac{g}{2}}\right)+2 \log g .
$$

This follows from Theorem (3) in [2] for $\theta(x)<x(1+1 /(2 \log x))$, together with $p_{n+1}<p_{n+2}<g$.

Shanks notes that for $g=10^{6}$, (1) gives

$$
p\left(10^{6}\right)<10^{5,565,715},
$$

while (2) gives

$$
p\left(10^{6}\right)<3.70 \cdot 10^{434,295} .
$$

Using Theorem (4) of [2], that $x-2.05 x^{1 / 2}<\theta(x)<x$ for $0<x<10^{8}$, and the exact primes $p_{n+1}=500,009, p_{n+2}=500,029$ from [3], we find that (5) gives

$$
p\left(10^{6}\right)<10^{217,160} \text {. }
$$

Thomas Parkin of Aerospace Corporation has kindly furnished the author with explicit computed values of $t$ and $A$ found by generating all the primes less than $5 \cdot 10^{5}$. He gives

$$
\begin{aligned}
\sum_{p \leqq 5 \cdot 10^{5}} \log _{10} p & =216851.104240, \\
t & =92,698,990,496, \\
A & =1.271 \cdot 10^{216,851},
\end{aligned}
$$

so that

$$
p\left(10^{6}\right)<10^{216,863} .
$$

Thus we can state that there is a string of at least one million consecutive composite numbers centered at $t A=92,698,990,496 \cdot \Pi p_{i}$ for $2 \leqq p_{i}<5 \cdot 10^{5}$.

RAND Corporation

Santa Monica, California

1. Daniel Shanks, "On maximal gaps between successive primes," Math. Comp., v. 18, 1964, pp. 646-650.

2. J. B. Rosser, \& Lowell Schoenfeld, "Approximate formulas for some functions of prime numbers," Illinois J. Math., v. 6, 1962, pp. 64-94. MR 25, *1139.

3. C. L. Baker \& F. J. Gruenberger, The First Six Million Prime Numbers, Microcard Foundation, West Salem, Wisconsin, 1959. 